\title{
Evaluation of the Teaching Quality of Physical Course Based on the Improved Technique for Order Preference by Similarity to an Ideal Solution*
}

\author{
Xiupeng $\mathrm{Gao}^{1}$ \\ Zhengzhou Normal University
}

\author{
Yong Zheng ${ }^{2}$ \\ China West Normal University
}

\begin{abstract}
As an important basic subject, physical teaching has been highly valued by students, schools and parents. Students begin to contact physical teaching from primary until graduating from the university. The good physical education can lay an excellent foundation for students' study, stimulate interest in study of other culture courses, and create conditions for further study. In order to improve the teaching quality of the physical course and find the deficiency in teaching, we evaluate the teaching quality of physical course. In order to improve the accuracy of the evaluation and to better evaluate the quality of physical teaching, we establish the quality evaluation index of physical teaching firstly. Then, we use the improved analytic hierarchy process to evaluate the teaching quality of the physical course. The evaluation results show that the accuracy and effectiveness of the proposed method.
\end{abstract}

\section{Keywords}

Teaching Quality • Physical Course • Topsis

\footnotetext{
*Results of China Sports General Sports Culture Development Center Base Project: A comparative study of basketball culture between China and the United States in the perspective of Globalization (15B005, 2015-2017).

${ }^{1}$ Zhengzhou Normal University, Zhengzhou 450000, China. Email: 419714140@qq.com

${ }^{2}$ Correspondence to: Yong Zheng. Physical Education Institute of China West Normal University, Nanchong 637009, China. Email: zhengyong7867@163.com 
Gao, Zheng / Evaluation of the Teaching Quality of Physical Course Based on the Improved Technique for Order ...

The "outline of curriculum reform in basic education" points out that it is necessary to establish the evaluation system for promoting the all-round development of students, the continuous improvement of teachers and the development of the curriculum. To evaluate the teaching quality of the physical course can learn the development of teaching quality of physical course, encourage students to study and improve learning quality. In order to promote the continuous improvement of the quality of the physical teaching, and correct the direction, we need to establish the physical course teaching evaluation system for promoting the all-round development of the students and teachers.

The basis of the evaluation study for the university physical course teaching was to promote the common development of teachers and students. Scholars evaluated it from teacher teaching and student learning. The scholars considered that we should adopt various forms during the evaluation process. We need combine the quantitative evaluation with the qualitative assessment. In addition, we also combined the others evaluation with the self-evaluation etc. then, it would realize the function of course teaching evaluation and promote the common development of teachers and students. Under the background of the new curriculum reform, some scholars have carried on the appraisal research to the university physical course teaching (Tan, 2012). The research held that it is beneficial to reflect on the theoretical model and practical operation of the current physical course teaching evaluation to establish the theory of constructivism. The classroom teaching evaluation had the great significance to stimulate the rapid development of students, teachers and schools.

Some scholars took the excellent physical teachers as the object of study and tracking evaluation of the teaching subject (O'Neil \& Boyce, 2018). Evaluation tables compiled by scholars referred to the standards of qualified teachers and the excellent teachers' standards formulated by the United States NBPTS. Then, they listened to the basic requirements of front-line physical teachers and middle school students in the course teaching. The purpose of the experiment was to test the scientific nature of the constructed evaluation plan.

Other scholars focused on the evaluation of physical education textbooks (Chatoupis, 2018). On the basis of summing up the previous theories and experiences, the scholars put forward the steps to establish the evaluation index system of middle school. After that, the scholars evaluated different editions of physical textbooks and pointed out the advantages and disadvantages of each edition of physical textbooks. In addition, many scholars have also studied it (SueSee, Pill \& Edwards, 2018; Linker \& Woods, 2018; Zhu, Haegele \& Davis, 2018).

To evaluate the teaching quality of physical course not only needed to establish the reasonable evaluation index, but also select the high precision method. AHP (Bian, Hu \& Deng, 2017) and Technique for Order Preference by Similarity to an Ideal Solution (TOPSIS) were commonly used evaluation methods. At the same time, AHP and TOPSIS can be well integrated together (Zyoud \& Fuchs-Hanusch, 2017). AHP-TOPSIS has widely applied to the industry (Karahalios, 2017), traffic management (Goyal \& Kaushal, 2017), agriculture (Sindhu, Nehra \& Luthra, 2017) and Enterprise management (Sekhar, Patwardhan \& Vyas, 2015) etc.

In this paper, we establish the teaching quality evaluation index of physical course. Then, we put forward an improved TOPSIS method which is proposed by the entropy method, AHP and TOPSIS. The first part of this paper is the research background. The second part sets up the evaluation index of the teaching quality of physical course. The third part puts forward the improved TOPSIS method. The fourth part is the experiment 
and the last part is the conclusion.

\section{Evaluation index}

A reasonable evaluation index is needed to be established in order to evaluate the teaching quality of physical course. Carrying out the teaching quality evaluation of physical course scientifically and effectively can make the students develop better. At the same time, it can also promote the professional development of teachers. Generally speaking, the function of teaching quality evaluation of physical course includes guiding function, stimulating function and improving function. As for the guiding function, the change of evaluation indexes can make the teacher adjust and perfect the classroom teaching constantly. For motivation, teachers can find their strengths and weaknesses, and they find the key influencing factors. Furthermore, teachers can make up for deficiencies so as to maintain their enthusiasm in physical teaching. For the improvement of the function, the evaluation can feedback various information timely, promote teachers to have their own problems in teaching and effective adjustment.

The establishment of evaluation follows the principles:

1. Combination between the evaluation teaching with evaluation learning. In the course of physical study, teachers should pay attention to the study of physical course while teaching. Physical course has many experiments. When the teachers teach, the students should actively study knowledge. In addition, physical teachers should mobilize their enthusiasm for study, promote learning, show their views, and grasp the relevant means and habits.

2. Combination between the quantitative and qualitative. In the process of physical teaching, some indexes cannot be quantified, including students' interests, teachers' own views on education and so on. Therefore, we should combine the qualitative with quantitative methods.

3. Diversification principle. The evaluation of the teaching quality of the physical course should be designed with many indexes. It involves both teaching materials and teachers, students, schools and so on. In the evaluation, teachers can recognize their lack. Then, the students can understand their rights and responsibilities.

4. Motivation and orientation. Carrying out assessment activities is to assess the status quo as a foothold. The purpose of the assessment is to find out the deficiencies and improve the quality of teaching.

\section{The improved TOPSIS method}

$\alpha$ is the weight of the subjective weight. $\beta$ is the weight of the objective weight. $\boldsymbol{W}$ is the comprehensive weight. We use entropy method to obtain subjective weight. Information entropy is,

$$
H(x)=-\sum_{i=1}^{n} p\left(x_{i}\right) \ln p\left(x_{i}\right)
$$

Then we process the data for the dimensionless. 


$$
\begin{aligned}
& X_{i j}=\frac{D-x_{i j}}{d-x_{i j}} \\
& P_{i j}=x_{i j} / \sum_{i=1}^{m} x_{i j}
\end{aligned}
$$

And

$$
\begin{aligned}
P_{i j} & =x_{i j} / \sum_{i=1}^{m} x_{i j}=\frac{1}{m} \\
e_{j} & =k \ln m
\end{aligned}
$$

Where $k=1 / \ln m, 0 \leq e_{j} \leq 1$

the weight is

$W^{\prime}=\frac{1+\frac{1}{\operatorname{Inm}} \sum_{i=1}^{m} p_{i j} \operatorname{In} p_{i j}}{\sum_{k=1}^{n}\left(1+\frac{1}{\operatorname{Inm}} \sum_{i=1}^{m} p_{i j} \operatorname{In} n p_{i j}\right)}$

We use AHP method to get the objective weight $W^{\prime \prime}$.

Then, we make the random consistency test to obtain the objective weight $W^{\prime \prime}$ of the AHP method.

Therefore, Comprehensive weight is,

$$
\begin{aligned}
& W=\alpha W^{\prime}+\beta W^{\prime \prime} \\
& \alpha^{2}+\beta^{2}=1
\end{aligned}
$$

The goal programming matrix is,

$$
\left\{\begin{array}{l}
\sum_{i=1}^{m} \sum_{j=1}^{n} \alpha_{i j}\left(\alpha W^{\prime}+\beta W^{\prime \prime}\right) \\
\alpha^{2}+\beta^{2}=1 \\
\alpha, \beta \geq 0
\end{array}\right.
$$

Using the Lagrange conditional extremum principle, we can get $\alpha_{l}{ }^{*}$ and $\beta_{l}{ }^{*}$. We make the normalization and get,

$$
\left\{\begin{array}{l}
\alpha^{*}=\frac{\sum_{i=1}^{m} \sum_{j=1}^{n} a_{i j} w_{j}^{\prime \prime}}{\sum_{i=1}^{n} \sum_{s}^{m} a_{i j}\left(w_{j}^{\prime}+w_{j}^{\prime \prime}\right)} \\
\beta^{*}=\frac{\sum_{i=1}^{m} \sum_{j=1}^{n} a_{s t} w_{s}^{\prime}}{\sum_{i=1}^{m} \sum_{j=1}^{n} a_{i j}\left(w_{j}^{\prime}+w_{j}^{\prime \prime}\right)}
\end{array}\right.
$$

Therefore,

$w_{j}=\alpha^{*} w_{j}^{\prime}+\beta^{*} w_{j}^{\prime \prime}$

The weight of the first level index $u_{i}$ is, 
Final weight set $W_{e}$ is,

$$
\begin{aligned}
& w_{e i j}=w_{j} \cdot w_{j k} \\
& W_{e}=\left\{w_{e 11,} w_{e 12}, \mathrm{~L}, w_{e j k}\right\}
\end{aligned}
$$

Initial processing of raw indexes

High quality index:

$$
a_{i j}=\frac{X_{i j}-X_{j}^{-}}{X_{j}^{+}-X_{j}^{-}}
$$

Low quality index:

$$
a_{i j}=\frac{X_{j}^{+}-X_{i j}}{X_{j}^{+}-X_{j}^{-}}
$$

Due to $\mathrm{a}_{\mathrm{ij}} \in[0,1]$, We define the optimal scheme and the worst scheme for the finite scheme.

$$
\begin{aligned}
& A^{+}=[1,1, \mathrm{~L}, 1]_{1 \times m}, j=1,2, \mathrm{~L}, m \\
& A^{0}=[0,0, \mathrm{~L}, 0]_{1 \times m}, j=1,2, \mathrm{~L}, m
\end{aligned}
$$

The distance between the evaluation index and the optimal scheme and the worst scheme is,

$$
\begin{aligned}
& D_{i}^{+}=\sqrt{\sum_{j=1}^{m} w_{j}\left(1-a_{i j}\right)^{2}} \\
& D_{i}^{-}=\sqrt{\sum_{j=1}^{m} w_{j}\left(0-a_{i j}\right)^{2}}
\end{aligned}
$$

We establish two dimensional space $\left(D_{i}^{+}, D_{i}^{-}\right)$for evaluating objects. We assume that $D_{i}^{+}=1 . D_{i}^{-}=1$ is the optimal reference point $\mathrm{A}$. Then, We compute the relative distance between the evaluated object and the reference point $\mathrm{A}$.

$$
C_{i}=\sqrt{\left[D_{i}^{+}-0\right]^{2}+\left[D_{i}^{-}-1\right]^{2}}
$$

We order the evaluation object from big to small according to the value of $C_{i}$. If the distance between the evaluated object $X_{i}, X_{j}(\mathrm{i} \neq \mathrm{j})$ and the reference point is equal, we set $(0,0)$ as suboptimal reference point B. Then we calculate the distance from the evaluation object $X_{i}, X_{j}(\mathrm{i} \neq \mathrm{j})$ to the reference point $\mathrm{B}$.

$$
C_{i}=\sqrt{\left[D_{i}^{+}-0\right]^{2}+\left[D_{i}^{-}-0\right]^{2}}
$$

We take the evaluation object that the value is smaller of $C_{i}$ as the better.

\section{Experiment}

After putting forward the teaching quality evaluation indexes of physical course and the improved analytic hierarchy process (AHP), we evaluate the teaching quality of physical courses in 4 middle schools. We use the entropy method and AHP method to obtain the objective weights and subjective weights. Then we calculate the 
Gao, Zheng / Evaluation of the Teaching Quality of Physical Course Based on the Improved Technique for Order ...

comprehensive weights.

The weight of teaching materials is 0.15 . Student learning is 0.21 . Classroom teaching is 0.23 . Student selfassessment is 0.18 . Otherwise is 0.09 .

The weights of breadth and depth of knowledge, teaching material structure and exercises of teaching materials are $0.42,0.38$ and 0.2 .

The weights of emotional states, learning style, participation level and learning effect of student learning are $0.28,0.23,0.18$ and 0.31 .

The weights of teaching thinking, teaching target, content of courses, teaching method, teaching process and culture of teaching of classroom teaching are $0.17,0.14,0.16,0.18,0.14$ and 0.21 .

The weights of love of physical, self-regulated learning ability and an appraisal of the physical teacher of student self-assessment are $0.39,0.35$ and 0.26 .

The weights of faculty, award situation and teaching style of otherwise are 0.42, 0.38 and 0.2.

The evaluation results are shown in the following table.

Table 1

\begin{tabular}{lcc}
\multicolumn{3}{c}{ Indicators for Analysis } \\
\hline School & $C_{i}$ & Ranking \\
\hline A & 0.5163 & 2 \\
B & 0.5785 & 1 \\
C & 0.4910 & 3 \\
D & 0.4756 & 4 \\
\hline
\end{tabular}

\section{Conclusion}

Evaluating the teaching quality of physical course, we can find out the shortcoming of physical course teaching and improve the quality of teaching. Physical is the basis of studying many other subjects well. A good physical course can lay a solid foundation for future work. In order to study the assessment of the quality of physical courses and improve the accuracy of evaluation, we establish the appropriate indicators. The indicators include 6 second level indicators and 23 thrid level indicators. Then, we propose an improved TOPSIS method. The method obtains the comprehensive weights by the entropy method and AHP method. Then it combines with analytic hierarchy process (AHP). Finally, we get the improved TOPSIS method. Experimental results show the accuracy and effectiveness of the proposed method.

\section{References}

Bian, T., Hu, J. T., \& Deng, D. (2017). Identifying influential nodes in complex networks based on AHP. Physica A: Statistical Mechanics and its Applications, 479, 422-436. https://dx.doi.org/10.1016/j.physa.2011.09.017

Chatoupis, C. C. (2018). Physical education teachers' use of Mosston and Ashworth's teaching styles: A 
Gao, Zheng / Evaluation of the Teaching Quality of Physical Course Based on the Improved Technique for Order ...

literature review. Physical Educator-US, 75(5), 880-900. https://dx.doi.org/10.18666/TPE-2018-V75-I58292

Goyal, T., \& Kaushal, S. (2017). An intelligent scheduling scheme for real-time traffic management using Cooperative Game Theory and AHP-TOPSIS methods for next generation telecommunication network. Expert Systems with Applications, 86, 125-134. https://dx.doi.org/10.1016/j.eswa.2017.05.071

Karahalios, H. (2017). The application of the AHP-TOPSIS for evaluating ballast water treatment systems by ship operators. Transportation Research Part D: Transport and Environment, 52, 172-184. https://dx.doi.org/10.1016/j.trd.2017.03.001

Li, Y. Q. (2010). Research and practice of classroom teaching assessment based on excellent physical teachers. Zhejiang Normal University, Curriculum and instruction theory (Physical Pedagogy).

Linker, J. M., \& Woods, A. M. (2018). "Like, we don't want to be PE teachers:" preservice classroom teachers' beliefs about physical education and willingness to incorporate physical activity. Physical Educator-US, 75(1), 77-98. https://dx.doi.org/10.18666/TPE-2018-V75-I1-7640

O'Neil, K., \& Boyce, B. A. (2018). Improving teacher effectiveness in physical education teacher education through field-based supervision. Physical Educator-US, 75(5), 835-849. https://dx.doi.org/10.18666/TPE2018-V75-I5-7739

Sekhar, C., Patwardhan, M., \& Vyas, V. (2015). A Delphi-AHP-TOPSIS based framework for the prioritization of intellectual capital indicators: a SMES perspective. Procedia - Social and Behavioral Sciences, 189, 275284. https://dx.doi.org/10.1016/j.sbspro.2015.03.223

Sindhu, S., Nehra, V., \& Luthra, S. (2017). Investigation of feasibility study of solar farms deployment using hybrid AHP-TOPSIS analysis: Case study of India. Renewable and Sustainable Energy Reviews, 73, 496511. https://dx.doi.org/10.1016/j.rser.2017.01.135

SueSee, B., Pill, S., \& Edwards, K. (2018). Interrogating assumptions of a curriculum: Queensland senior physical education syllabus. Physical Educator-Us, 75(5), 850-879. https://dx.doi.org/10.18666/TPE-2018V75-15-8283

Tan, S. H. (2012). Research on classroom teaching evaluation of senior middle school physical under the new curriculum reform. Shaanxi Normal University, Subject teaching (Physical).

Zhu, X. H., Haegele, J. A., \& Davis, S. (2018). Physical Educators' Habitual Physical Activity and Self-Efficacy for Regular Exercise. Physical Educator-US, 75(1), 50-63, https://dx.doi.org/10.18666/TPE-2018-V75-I17675

Zyoud, S. H., \& Fuchs-Hanusch, D., 2017, A bibliometric-based survey on AHP and TOPSIS techniques. Expert Systems with Applications, 78, 158-181. https://dx.doi.org/10.1016/j.eswa.2017.02.016 